\title{
Site-Directed Mutagenesis and Generation of Chimeric Viruses by Homologous Recombination in Yeast to Facilitate Analysis of Plant-Virus Interactions
}

\author{
Delin Liang, ${ }^{1}$ Stewart M. Gray, ${ }^{1}$ Igor Kaplan, ${ }^{1}$ and Peter Palukaitis ${ }^{2}$ \\ ${ }^{1}$ United States Department of Agriculture-Agricultural Research Service and Department of Plant Pathology, Cornell \\ University, Ithaca, NY 14850, U.S.A.; ${ }^{2}$ Scottish Crop Research Institute, Invergowrie, Dundee DD2 5DA, U. K.
}

Submitted 15 September 2003. Accepted 30 January 2004.

\begin{abstract}
A yeast homologous recombination system was used to generate mutants and chimeras in the genome of Potato leafroll virus (PLRV). A yeast-bacteria shuttle vector was developed that allows mutants and chimeras generated in yeast to be transformed into Escherichia coli for confirmation of the mutations and transformed into Agrobacterium tumefaciens to facilitate agroinfection of plants by the mutant PLRV genomes. The advantages of the system include the high frequency of recovered mutants generated by yeast homologous recombination, the ability to generate over 20 mutants and chimeras using only two restriction endonuclease sites, the ability to introduce multiple additional sequences using three and four DNA fragments, and the mobilization of the same plasmid from yeast to $E$. coli, A. tumefaciens, and plants. The wild-type PLRV genome showed no loss of virulence after sequential propagation in yeast, $E$. coli, and $A$. tumefaciens. Moreover, many PLRV clones with mutations generated in the capsid protein and readthrough domain of the capsid protein replicated and moved throughout plants. This approach will facilitate the analysis of plant-virus interactions of in vivo-generated mutants for many plant viruses, especially those not transmissible mechanically to plants.
\end{abstract}

The analysis of plant-virus interactions has been facilitated considerably by the development of biologically active cDNA clones and the ability to exchange genome segments as well as generate site-directed mutations in such cDNAs. In the case of viruses with positive-sense RNA genomes, techniques have been developed to confer infection from biologically active cDNA clones (Boyer and Haenni 1994). These involve inoculation with either infectious transcripts from the cDNA synthesized in vitro or plasmids containing the viral genomic sequences flanked by transcription promoter and termination sequences, usually derived from the DNA virus Cauliflower mosaic virus (CaMV). In many cases, viruses or viral genomes cannot be transmitted to plants by mechanical inoculation (Hull 2002). However, infections have been established by the use of an inoculation technique called agroinfection, in which a disarmed plasmid, the T-DNA, containing viral sequences flanked by transcription regulatory sequences, is introduced into plant cells by the bacterium Agrobacterium tumefaciens, and the viral

Corresponding author: P. Palukaitis; Telephone: +44 1382 568523; Fax: +44 1382 562426; E-mail: ppaluk@scri.sari.ac.uk genomes are expressed in the inoculated cells (Grimsley et al. 1986; Leiser et al. 1992; Nurkiyanova et al. 2000; Prüfer et al. 1995). Agroinfection also occurs in vascular cells, because plants have been agroinfected with infectious cDNAs of phloem-limited viruses (such as poleroviruses) and progressive infections have occurred (Kawchuk et al. 2002; Leiser et al. 1992; Nurkiyanova et al. 2000; Prüfer et al. 1995). Due to the high efficiency of infection achieved by this technique, agroinfection also has been used with viroids and viruses that can be transmitted mechanically, but for which infection via some biologically active plasmids is not as efficient (Gardner et al. 1986; Lamprecht and Jelkmann 1997; Liu and Lomonossoff 2002; Liu et al. 2002; Saunders et al. 2002).

The techniques for exchange of viral sequences to generate chimeric viruses, or for the generation of site-directed mutants, have been developed for use with plasmids in Escherichia coli (Sambrook and Russell 2001). Such methods generally are not amenable for use with the much larger, lower-yielding plasmids required for T-DNA transfer from $A$. tumefaciens. Thus, mutagenesis of existing viral genomes involves a number of steps, and the transfer of viral genetic material to different plasmids for growth in various bacteria, for the different stages of the overall process. In addition, the location and number of particular restriction sites is often a limitation to the generation of specific site-directed mutants.

We present here an approach to the generation of mutants and chimeras that reduces these limitations and the number of steps needed to modify the viral genomes and transfer of infectious viral DNAs prior to agroinfection. In this approach, elements of the yeast (Saccharomyces cerevisiae) homologous recombination system have been incorporated into an Agrobacterium vector to facilitate segment exchange, deletions, and site-directed mutagenesis of infectious virus clones. We present data using the polerovirus Potato leafroll virus (PLRV).

\section{RESULTS}

Strategy for generation

of mutant PLRV clones suitable for agroinfection.

The approach used to generate mutants of a biologically active cDNA clone of PLRV involved three steps: i) preparation of a full-length cDNA clone of the PLRV genome, flanked by $35 \mathrm{~S}$ RNA promoter and terminator sequences, both derived from $\mathrm{CaMV}$, in an E. coli-A. tumefaciens shuttle vector, pBIN19; ii) introduction of a sequence encoding a yeast origin of replication (from the yeast $2-\mu \mathrm{m}$ DNA) plus a selectable 
marker into the pBIN19 shuttle vector containing the fulllength PLRV insert, to allow replication of the plasmid in yeast cells; and iii) the construction of PLRV mutants by homologous recombination in yeast, which then can be transformed into A. tumefaciens for agroinfection. This approach does not require any further transfer of the PLRV genomic

pBNUP110 19kbp

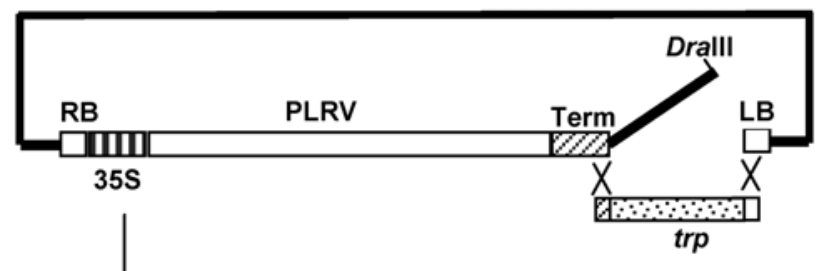

Yeast transformation

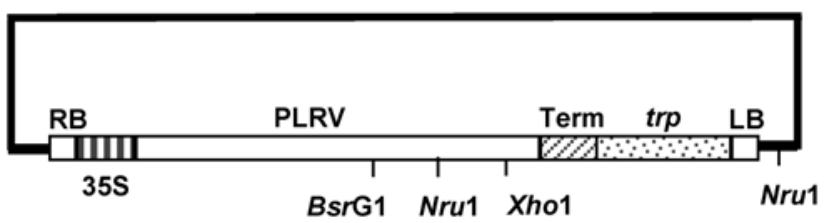

Fig. 1. Schematic diagram representing the generation of the yeast-bacteria shuttle vector $\mathrm{pBPY}$. The plasmid pBNUP110 contains a full-length cDNA clone of the genome of Potato leafroll virus (PLRV), flanked by a 35S RNA promoter region (35S) and a transcription termination sequence (Term), both derived from Cauliflower mosaic virus, inserted in the plasmid pBIN19, an Escherichia coli-Agrobacterium tumefaciens shuttle vector. pBNUP110 was linearized by digestion with DraIII, and modified further by insertion of the yeast 2- $\mu$ m DNA and trp gene from the plasmid pJG4-5 (trp), using homologous recombination in yeast. The resulting plasmid, pBPY, replicated in yeast and retained the ability to replicate in E. coli and A. tumefaciens. LB and RB are the left border and right border, respectively, of the T-DNA. Note that some sequences of pBNUP110 (between Term and LB) are lost after recombination.
cDNA to other plasmid vectors prior to inoculation of plants, although transfer of the plasmids back to E. coli was used to facilitate sequence verification of the generated mutants and estimation of the recombination efficiency.

A full-length cDNA clone of PLRV previously had been transferred to pBIN19, to produce pBNUP110 (Fig. 1) for use in agroinfection and transformation (Franco-Lara et al. 1999; Nurkiyanova et al. 2000), obviating the need for the first step here. A DNA fragment containing the yeast 2- $\mu$ m DNA plus trp gene selectable marker was amplified by polymerase chain reaction (PCR) and introduced into the T-DNA of pBNUP110 close to the left border by recombination in yeast (Fig. 1). There were 25- and 23-bp overlaps between the PCR termini and sequences of PBNUP110. This yielded the plasmid pBPY (Fig. 1), which was able to replicate in yeast. Yeast cells auxotrophic for tryptophan also appeared when only the PCR fragment containing the 2- $\mu$ m DNA plus trp gene was used for transformation, although in considerable fewer numbers $(900$ versus 100 colonies, respectively). Approximately two-thirds of the plasmids examined (11 of 15) contained the $2-\mu \mathrm{m}$ DNA plus trp gene sequences in the T-DNA of pBNUP110 (Fig. 1). Thus, any virus clones developed for agroinfection can be converted into a form that can replicate in yeast.

Transformation of pBPY into A. tumefaciens and agroinfection of Nicotiana clevelandii and $N$. benthamiana consistently have yielded infection by PLRV in 80 to $90 \%$ of the plants, a level similar to agroinfection by $A$. tumefaciens containing pBNUP110 (Lee et al. 2002). There was no difference in the symptoms produced by pBPY versus pBNUP110 in these plant species, and the virus titers measured in uninoculated leaves by enzyme-linked immunosorbent assay (ELISA) were similar (data not presented).

\section{Construction of PLRV mutants and chimeras by recombination.}

Digestion of pBPY to remove the DNA fragment to be replaced and dephosphorylation of the ends yielded a linear plasmid unable to replicate in yeast, unless the plasmid was again circularized. Circularization was facilitated by recombination with overlapping DNA fragments, generating various

Table 1. Mutated genomes of Potato leafroll virus (PLRV) generated by homologous recombination in yeast

\begin{tabular}{|c|c|c|c|c|c|}
\hline Mutants & No. of fragments ${ }^{a}$ & $\begin{array}{l}\text { Overlap in vector } \\
(\mathbf{b p})^{\mathbf{b}}\end{array}$ & $\begin{array}{l}\text { Overlapping sequences } \\
(\text { (bp) })^{\mathbf{c}}\end{array}$ & Transformation ratio ${ }^{\mathrm{d}}$ & Recombinants detected $^{\mathrm{e}}$ \\
\hline $2-\mu \mathrm{m}$ DNA & 2 & 6,309 & 25,23 & $9: 1$ & $11 / 15$ \\
\hline$\Delta \mathrm{RTD}^{\mathrm{f}}$ & 3 & 0,0 & $70,48,382$ & $80: 1$ & $1 / 1$ \\
\hline$\Delta 3-\mathrm{RTD}^{\mathrm{g}}$ & 3 & 0,0 & $70,46,382$ & $20-60: 1$ & $42 / 42^{\mathrm{h}}$ \\
\hline RPV-CP ${ }^{\mathrm{i}}$ & 2 & 29,336 & 28,21 & $7: 1$ & $1 / 1$ \\
\hline RPV-RTD ${ }^{\mathrm{j}}$ & 2 & $1,192,256$ & 25,25 & $3: 1$ & $3 / 5$ \\
\hline RPV-CPRTD ${ }^{k}$ & 2 & 29,256 & 28,25 & $7: 1$ & $1 / 1$ \\
\hline $\mathrm{GFP}^{\mathrm{l}}$ & 2 & 284,256 & 24,24 & $5: 1$ & $3 / 5$ \\
\hline IRES-GFP $^{\mathrm{m}}$ & 4 & 0,256 & $70,23,24,24$ & $5: 1$ & $3 / 5$ \\
\hline
\end{tabular}

${ }^{a}$ Number of DNA fragments (polymerase chain reaction [PCR] products plus vector DNA) combined for transformation into yeast.

${ }^{b}$ Number of base pairs to overlap in vector: the distance between the sites in the vector DNA that overlap with the PCR fragments and the restriction endonuclease sites in the linearized vector DNA.

${ }^{\mathrm{c}}$ Number of base pairs overlapping between the PCR products, and between the PCR products and the vector, upstream to downstream (left to right) of the restriction endonuclease sites.

${ }^{\mathrm{d}}$ Ratio of colonies observed on plates transformed with linearized and dephosphorylated vector plus PCR products (recombinants) vs. linearized and dephosphorylated vector alone.

${ }^{\mathrm{e}}$ Number of clones found to contain the specified mutant over number of clones analyzed.

${ }^{\mathrm{f}} \triangle \mathrm{RTD}=$ deletion of sequences in the PLRV capsid protein (CP) readthrough domain (RTD).

$\mathrm{g} \triangle 3$-RTD $=14$ different mutants of PLRV containing three amino acid-deletions in the RTD.

${ }^{\mathrm{h}}$ Three clones were screened for each of the 14 RTD mutants, with each clone containing the expected mutation.

${ }^{\mathrm{i}}$ Replacement of the CP open reading frame (ORF) of PLRV with the CP ORF of Cereal yellow dwarf virus (CYDV), RPV strain.

${ }^{j}$ Replacement of RTD ORF of PLRV with the RTD ORF of CYDV-RPV.

${ }^{k}$ Replacement of the both CP and RTD ORFs of PLRV with those of CYDV-RPV.

${ }^{1}$ Replacement of sequences encoding the C-terminus of the PLRV RTD with the gene encoding the green fluorescent protein (GFP).

${ }^{\mathrm{m}}$ Replacement of sequences encoding the C-terminus of the PLRV RTD with the internal ribosome entry site (IRES) of Rhopalosiphum padi virus and the gene encoding the GFP. 
mutant and chimeric PLRV sequences (Table 1). To generate the mutants, linearized pBPY was combined with one to three overlapping PCR fragments representing the mutated region or heterologous sequences (Fig. 2). In the first case ( $\triangle$ RTD), the two fragments contained both i) deletions of $100 \mathrm{bp}$ of PLRV sequences (nucleotides 4,216 to 4,315), shown to be important for the readthrough of the termination signal of the open reading frame (ORF) encoding the capsid protein (CP) (ORF 3), normally allowing expression of ORF5, which encodes the readthrough domain (RTD) (Bruyère et al. 1997), and ii) introduction of additional terminator sequences after the CP ORF. This translational mutant led to the generation of a PLRV that produced CP only, and no RTD. Analysis of plant tissue agroinfiltrated with the mutant $\triangle$ RTD showed the presence of PLRV CP, but not of the RTD (Fig. 3A). Reverse transcription (RT)-PCR and sequence analysis of the progeny viral RNAs confirmed that the mutations introduced were maintained (Fig. 3B and data not shown). These results are consistent with previous observations made with mutants of the polerovirus Beet western yellows virus (BWYV), in which it was shown that a mutant comparable to $\triangle \mathrm{RTD}$ was able to replicate, form virus particles, and move throughout the plant (Bruyère et al. 1997). A similar approach was used to generate a series of deletions of three consecutive codons in the sequences encoding the RTD ( $\Delta 3$-RTD) (Table 1).

To illustrate the utility of this mutagenesis approach, two other types of alterations of the PLRV genome were generated: i) replacement of a fragment of the PLRV genome by some heterologous gene (e.g., replacement of the CP or RTD of PLRV by those of the polerovirus Cereal yellow dwarf virus, RPV strain [CYDV-RPV] [Fig. 2, RPV-CP, RPV-RTD, RPVCPRTD] or, similarly, replacement of part of the PLRV RTD [nucleotides 5,202 to 5,763] by sequences encoding the green fluorescent protein [GFP] [Fig. 2, GFP]); and ii) insertion of multiple heterologous genes into the PLRV sequence using three PCR fragments (Fig. 2, IRES-GFP). In all cases, mutants were generated readily, although, in general, the ratio of yeast colonies obtained for the recombinants versus the linearized vector background was much lower for the mutants involving introduction by recombination of a single PCR fragment (Table 1). In most cases, analysis of only a single colony was required to detect the expected mutant (Table 1 and data not shown). The exception was the recombination involving the vector and three PCR fragments, which introduced the sequences encoding the GFP and an upstream internal ribosome entry site (IRES) from Rhopalosiphum padi virus (RhPV) (Domier and McCoppin 2003) (Fig. 2, IRES-GFP). This construct also showed a lower transformation ratio (Table 1). These lower ratios most likely were due to the fewer overlapping nucleotides between the PCR fragment and the vector, or to the fact that the recombination sites in the chimera were not proximal to the restriction endonuclease sites in the vector (Table 1; discussed below).

The GFP construct was infectious and expressed the expected CPRTD-GFP fusion protein (Fig. 4B), and GFP was observed in agroinfiltrated plant tissue (Fig. 4C). In contrast, the IRES-GFP construct did not result in the translation or expression of GFP in vivo (Fig. 4B and C), although the expected mutant was recovered from the recombination event (Fig. 4A and data not shown) and the virus did replicate and accumulate CP similar to wild-type virus (WT) (Fig. 4B). It is likely that the RhPV IRES sequence does not function as expected in planta.

\section{Optimization of frequency of recombination.}

Previous studies have demonstrated that the recombination frequency increased with increasing length of overlapping
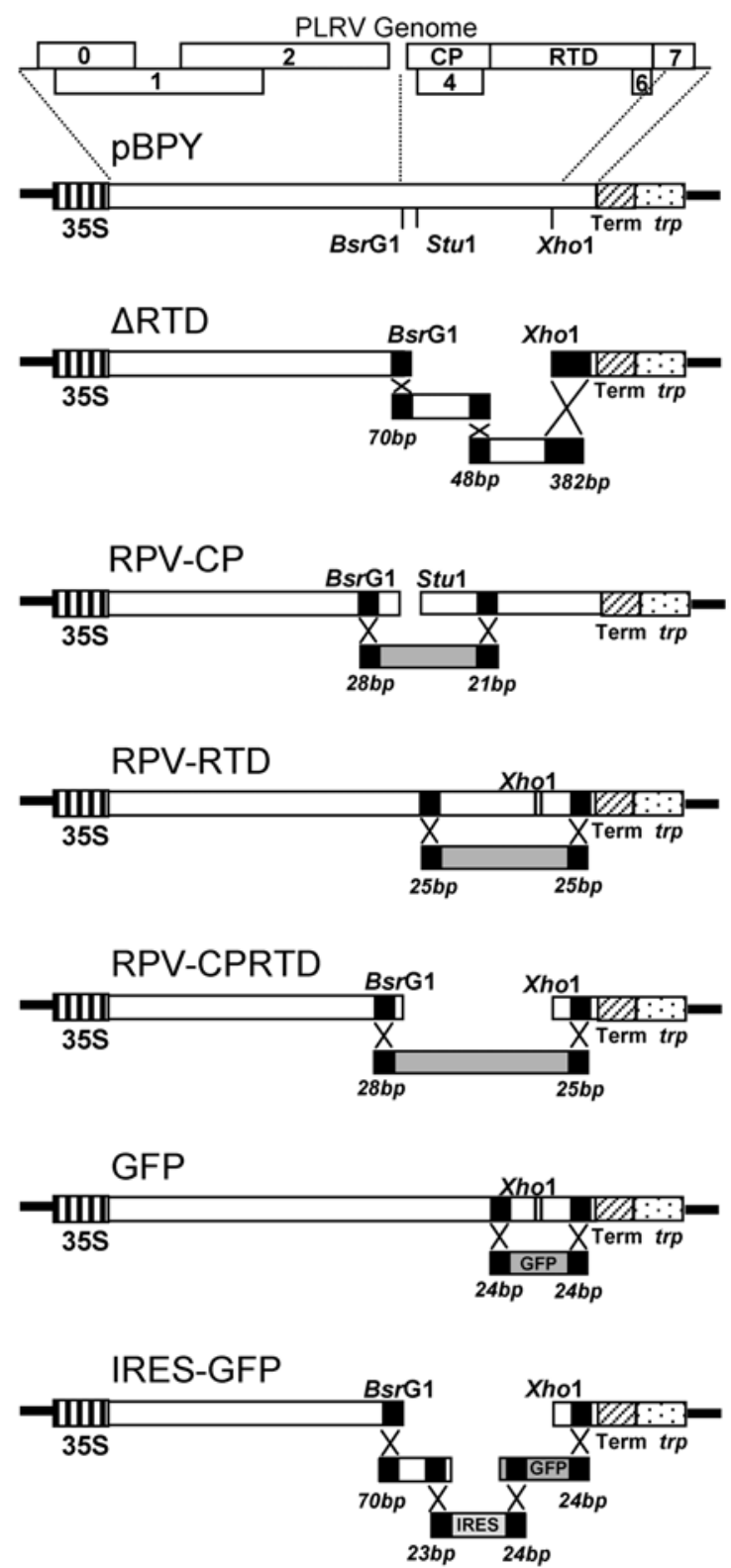

Fig. 2. Schematic representation of DNA mutagenesis and chimeric insertion of the structural genes of Potato leafroll virus (PLRV) using the yeast homologous recombination system. The plasmid pBPY was digested with one or a combination of the restriction endonucleases BsrGI, Stu1, or XhoI, to linearize the plasmid or remove the part of open reading frames (ORFs) encoding the capsid protein (CP) (ORF3) and the readthrough domain (RTD) (ORF5). After dephosphorylation, the remaining plasmid, together with overlapping DNA fragments containing the desired sequences or mutations, generated by the polymerase chain reaction (PCR), were transformed into yeast. The black-filled boxes represent overlapping regions of DNA and sites of recombination. The size, in base pairs (bp), of the overlapping region is provided below each black-filled box. In the $\triangle$ RTD mutant, the overlapping sequence of the two PCR products was constructed to insert an additional stop codon at the $3^{\prime}$ end of ORF3, and delete 100 nucleotides at the $5^{\prime}$ end of ORF5, also causing a frameshift that prevented the translation of the readthrough protein. In the RPV-CP, RPV-RTD, and RPV-CPRTD mutants, ORF3, ORF 5, and ORF3 plus ORF5, respectively, of PLRV were replaced with the homologous sequences from Cereal yellow dwarf virus (CYDV)-RPV. In the green fluorescent protein (GFP) mutant, a portion of the ORF5 was replaced with the GFP. In the internal ribosome entry site (IRES)-GFP mutant, the PLRV ORF5 was replaced with the IRES of Rhopalosiphum padi virus fused in-frame with the gene encoding GFP. A map of the PLRV genome is provided to orientate the reader to the area being mutated or exchanged. The approximate locations of the $B s r G I$ and XhoI restriction sites are indicated by the dotted lines. All of the fragments depicted in the figure are not exactly to scale. 
sequences (Manivasakam et al. 1995; Oldenburg et al. 1997; Raymond et al. 1999). However, in the examples given here, the mutants were not generated using different lengths of overlapping DNAs in a manner that would facilitate a direct comparison. The recovery of mutants is influenced by two factors: the extent of overlap (in base pairs) between the PCR fragments and the vector, and the distance between the regions of overlap and the site of cleavage in the vector (Table 1; Fig. 2). Thus, those mutants ( $\triangle \mathrm{RTD}$ and $\Delta 3$-RTD) generated using PCR fragments that overlapped at their ends with the vector by 70 and 382 bp were produced with the highest apparent recombination frequency (Table 1). In both of these cases, the overlapping positions of the PCR fragments with the vector were located adjacent to the restriction endonuclease sites (Table 1; Fig. 2). In contrast, those mutants generated with DNA fragments that overlapped the vector by 24 and $25 \mathrm{bp}$, where the overlapping positions were located a considerable distance from the restriction endonuclease sites (e.g., RPVRTD and GFP in Table 1), showed a much lower apparent recombination frequency. In addition, mutants (RPV-CP and RPV-CPRTD in Table 1) in which one end of the overlapping sequences with the vector was in close proximity (29 bp) to the restriction endonuclease site showed a higher apparent recombination frequency than when both ends were hundreds of base pairs from the sites of recombination (RPV-RTD and GFP in Table 1). This was true even though the number of base pairs of overlap between the PCR fragment and the vector were comparable ( 21 to 28 bp and 25 to 28 bp versus 25 and $24 \mathrm{bp}$ for the above four examples, respectively). Moreover, we have obtained sufficient recombination to readily detect re-

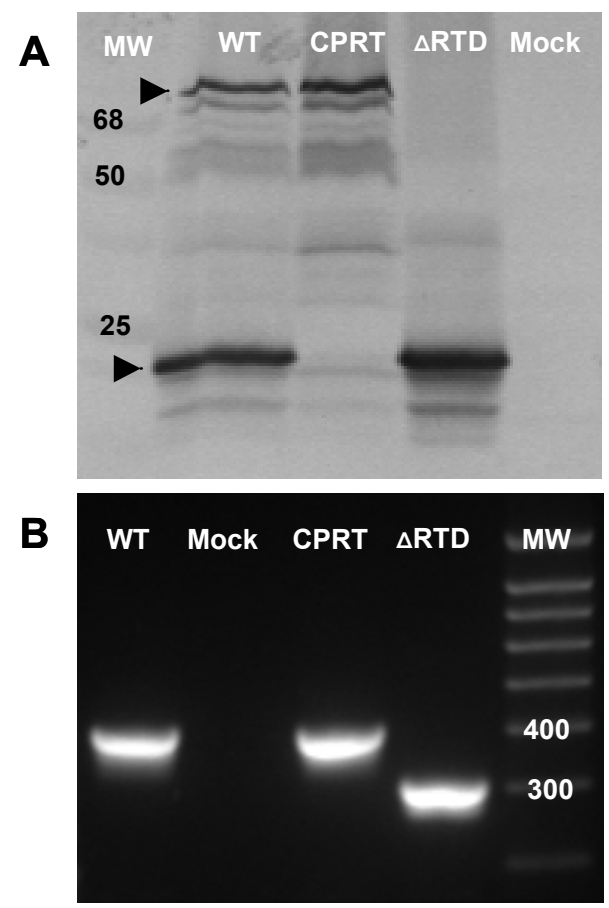

Fig. 3. A, Western blot analysis and $\mathbf{B}$, reverse-transcription polymerase chain reaction (RT-PCR) for wild-type (WT) Potato leafroll virus (PLRV), mock inoculated, PLRV with an altered stop codon in open reading frame (ORF) 3 (coat protein, CPRT), and PLRV that does not express readthrough protein due in part to a 100-nucleotide deletion in ORF5 $(\triangle \mathrm{RTD})$. Total protein or total RNA was isolated from Nicotiana benthamiana leaves agroinfiltrated with the virus constructs 6 days prior. Virus proteins were detected using an anti-PLRV coat protein monoclonal antibody. Arrowheads identify the $22-\mathrm{kDa}$ coat protein and approximately $80-\mathrm{kDa}$ readthrough protein. PCR products were amplified using PLRVspecific primers that generate a 391-bp fragment that spans the ORF3ORF5 junction. combinant clones with as few as 20 bp of overlapping DNAs (data not shown). Thus, in the case of the 14 three-amino acid deletion mutants in the RTD (data not shown), as well as the 100-nucleotide (nt) deletion in the RTD, it was not necessary to screen the positive clones, but simply to take one colony at random and verify the nature of the deletion mutant. This is because there were 20 to 80 times more colonies on the recombinant plates than on the control plates (Table 1). In the case of the chimeras formed between PLRV and either CYDV-RPV or the GFP encoding sequences, several clones needed to be chosen at random to make sure that at least one of these would contain the expected chimera (Table 1). This also was true of the chimera formed with sequences encoding the GFP and an IRES, where four DNA fragments were involved in the recombination event (Table 1), and where only one fragment showed extensive overlap (70 bp) with the sequences adjacent to one of the restriction endonuclease sites in the vector (Fig. 2E).

Randomly picking three $E$. coli colonies for each of $14 \mathrm{mu}-$ tants with three-amino acid deletions in the RTD, followed by PCR and sequencing analysis, showed that each colony contained the desired mutation. However, when only one colony was picked randomly from each of the 14 mutants, 12 of the 14 mutants had the expected mutation, but two colonies had 1 or $3 \mathrm{nt}$ missing from the region of site-directed mutagenesis (data not shown). These unexpected mutations, which occurred at a low frequency, could have been due to contamination by a low level of faulty primer (as was verified in one case), a PCR error, or illegitimate recombination in yeast. Nevertheless, this example shows that the vast majority of the yeast cells analyzed contained the expected mutants.

\section{DISCUSSION}

In yeast, homologous recombination is an important strategy for repair of double-stranded DNA breaks and creating genetic diversity (Orr-Weaver and Szostak 1985; Sung et al. 2000). Overlapping DNA fragments undergo recombination readily when co-transformed into yeast cells (Botstein and Shortle 1985; Kunes et al. 1985, 1987; Ma et al. 1987; Raymond et al. 1999). Several cloning methods have been reported, based on yeast homologous recombination (Gunyuzlu et al. 2001; Oldenburg et al. 1997; Raymond et al. 1999, 2002). However, most of these methods focus on gene cloning but not mutagenesis. Dun $\varnothing$ and associates (1999) developed a method for mutagenesis by double-stranded oligonucleotide replacement to generate deletions. However, this method depends on the accessibility of unique restriction enzyme sites in the plasmid. The in vivo site-directed mutagenesis method of Storici and colleagues (2001), called "delitto perfetto", has more flexibility and is very efficient, but requires the introduction of a counter-selectable reporter cassette. More recently, an efficient site-directed mutagenesis method was described for a small plasmid using a three-fragment homologous recombination system in yeast (Kitagawa and Abdulle 2002). The method described here for making mutants in the genome of PLRV in a large and low-copy-number plasmid also is very efficient, and uses homologous recombination in yeast with two or three DNA fragments in a plasmid that can be grown in yeast and then used for agroinfection. We have shown that not only sitedirected mutagenesis but also precise deletions and chimeric fragments could be introduced into the PLRV genome with just one cloning step. In addition to the high frequency of mutants recovered after recombination in yeast and the use of a single shuttle vector for three different organisms, another advantage of the system is the ability to generate over 20 mutants and chimeras with only two restriction enzyme sites in the DNA corresponding to the viral genome. Moreover, because 
recombination also occurred several hundred base pairs from the restriction endonuclease sites, with concomitant removal of all intervening vector sequences, this recombination strategy is less dependent upon the proximity of appropriate restriction endonuclease sites than the traditional ligation-based approach.

The use of this system for mutagenesis in yeast and agroinfection using the plasmid obtained from yeast should be broadly applicable to most plant viruses, especially those with single-component genomes. This combination of methods may be particularly useful for mutagenesis and infection in cases where the infectivity of RNA transcripts from cDNA clones has proven to be extremely poor. (Satyanarayana et al. 2001). In addition to the generation of mutants that can be used to assess viral protein functions and the interactions of these proteins with plant components during infection or resistance, this approach also can be used to generate protein fusions with tags such as Myc, FLAG, and 6xHis. These could be used for subcellular localization studies, using antibodies to the tags, and for the affinity purification of host components associated with the tagged viral-encoded proteins, further facilitating a better understanding of the nature of plant-virus interactions. Finally, cDNA libraries could be inserted into a virus vector using the yeast recombination system described here, and those virus vectors could be used for viral-induced gene silencing, as has been done for the Tobacco rattle virus vector system, which also is introduced into plants via agroinfection (Liu et al. 2002). Such a system may facilitate the rapid functional screening of cDNA libraries.

\section{MATERIALS AND METHODS}

\section{Yeast and bacterial strains and plasmids.}

Plasmid pJG4-5, containing the yeast 2- $\mu \mathrm{m}$ DNA and a trp gene, as well as yeast strain RFY206 (MATatrp1 $\Delta$ ::hisGhis $3 \Delta$ 200ura3-52lys2 $\Delta$ 201leu2-3), were obtained from Origene Technologies Inc. (Rockville, MD, U.S.A.). Plasmid pBNUP110, containing the PLRV genome flanked by CaMV transcription regulatory signals in pBIN19, was described previously (Franco-Lara et al. 1999; Nurkiyanova et al. 2000). Purified CYDV-RPV RNA was used as a template for amplifying the CP, RTD, and CP-RTD by RT-PCR (Sambrook and Russell 2001) The primer design was based on the sequence of CYDVPRV (GenBank accession number NC004751). Plasmid pBIN35S-mGFP4 was used as a template for amplifying the GFP gene (accession number U87624) by the PCR. A plasmid containing the sequence representing the IRES from RhPV was obtained from L. Domier (Domier and McCoppin 2003). A. tumefaciens strain LBA-4404 was used for agroinfection and $E$. coli strain DH10B was used for selection of recombinant clones derived in yeast.

\section{Construction of a yeast-bacteria shuttle vector.}

The two primers 5'-CGAAAAACCGTCTATCAGGGCGAT GAAGGATGAG-ACTAATCCAA-3' and 5'-GCTAGAGGAT CCCCGGGTACCGAGGTTCCGCGC-ACATTTCCCCGA-3' were used to amplify a DNA fragment containing the $2-\mu \mathrm{m}$ DNA plus trp gene by the PCR from the plasmid pJG4-5 (accession number U89961). The 3' halves of each primer contained sequences for amplification of the 2- $\mu$ m DNA, whereas the $5^{\prime}$ halves of each primer contained sequences derived from the plasmid pBNUP110, required to facilitate the recombination. The plasmid pBNUP110 was linearized by digestion with DraIII, located in the T-DNA close to the left border sequence (Fig. 1), and dephosphorylated by treatment with calf intestinal alkaline phosphatase (New England Biolabs, Beverly, MA, U.S.A.), using standard procedures (Sambrook and Russell,
2001). The linearized, dephosphorylated pBNUP110 (100 ng) and the PCR fragment $(1 \mu \mathrm{g})$ were combined and transformed into yeast, essentially as described in the Duplex-A yeast twohybrid system manual (Origene Technologies). After 3 days, yeast colonies were pooled for total DNA extraction, as described in the manual. The pooled yeast DNA was transformed into E. coli strain DH10B and transformants were selected for resistance to kanamycin. Individual $E$. coli colonies were lysed and the DNA was analyzed by the PCR, amplifying the $2-\mu \mathrm{m}$ DNA plus trp gene. The presence of the $2-\mu \mathrm{m}$ DNA sequences in the pBIN19-derived plasmid was confirmed by digestion of the plasmid with $N r u$ I, yielding a 2.5-kbp fragment (Fig. 1). One such clone, designated pBPY, was used for all further manipulations.

\section{Construction of PLRV mutants and chimeras.}

Plasmid pBPY was digested with XhoI alone (position 5,482 in the PLRV genome), XhoI in combination with BsrGI (position 3,627 in the PLRV genome, 38 bp after the start of the CP ORF), or BsrGI in combination with StuI (positions 3,906 and
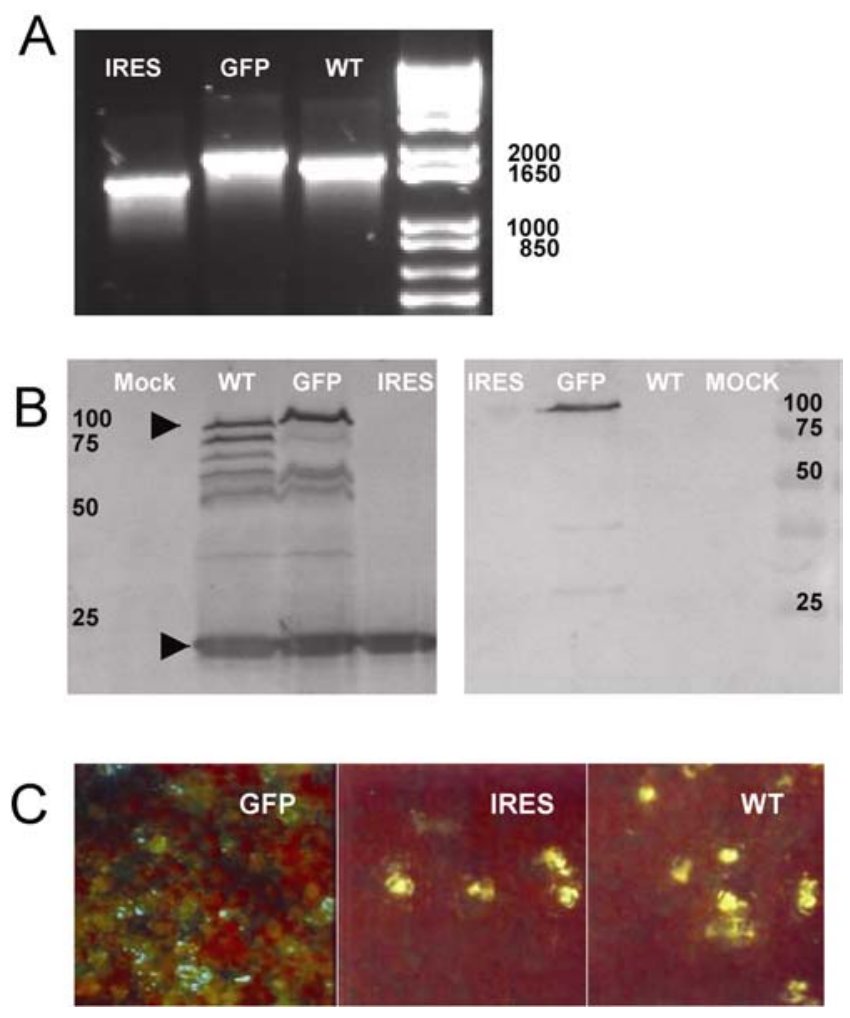

Fig. 4. A, Reverse-transcription polymerase chain reaction (RT-PCR), B, Western blot analysis, and C, fluorescence microscopy of Nicotiana benthamiana tissue agroinfiltrated with wild-type (WT) Potato leafroll virus (PLRV) and two mutants. In the green fluorescent protein (GFP) mutant, the C-terminal portion (nucleotides 5,203 to 5,738) of open reading frame (ORF) 5 was replaced in-frame with the gene encoding GFP (717 nucleotides). In the internal ribosome entry site (IRES)-GFP (IRES) mutant, the PLRV ORF5 (nucleotides 4,217 to 5,738) was replaced with the IRES of Rhopalosiphum padi virus (624 nucleotides) fused in-frame with the gene encoding GFP (717 nucleotides). RT-PCR products shown on the left and right halves of panel A were amplified using forward primers PLRV4159-79 and PLRV4823-45, respectively. The same reverse primer (PLRV 5855-83) was used in both reactions. Proteins observed on the left and right sides of panel B were detected with an anti-PLRV coat protein monoclonal antibody and an anti-GFP antibody, respectively. Arrowheads identify the $22-\mathrm{kDa}$ coat protein and approximately $80-\mathrm{kDa}$ readthrough protein. C, Cells infected with the GFP mutant express GFP protein in discrete areas within cells. Numerous dead cells were observed to autofluoresce (yellow fluorescence in IRES and WT) in all infiltrated tissues, but no GFP expression was observed in cells infected with WT or IRES. 
$3,957)$, followed by dephosphorylation of the plasmid. The digested plasmid was combined with various PCR fragments to generate mutants and chimeras, after co-transformation in yeast. The PCR fragments contained terminal sequences that overlapped with the linearized vector and, in the case of the mixtures of three and four fragments, also overlapped with each other to varying extents (Table 1; Fig. 2). The PCR fragments were generated using primers that corresponded to sequences present at, near (29 bp), or up to $1,192 \mathrm{bp}$ from the ends of the ORFs of the CP (nucleotides 3,589 to 4,215 in the PLRV genome) or RTD (ORF5; nucleotides 4,216 to 5,739). In the case of the chimeric CYDV-RPV constructs, the primers also contained the corresponding sequences of CYDV-RPV to allow amplification of the CYDV-RPV ORFs and recombination with overlapping identical sequences between the termini of the PCR products and the PLRV genome. For the 14 three-amino acid deletion mutant constructs, primers were altered in sequence, to delete 9 nt (three codons) in ORF5. For the GFP chimera constructs, the primers also contained the corresponding terminal sequences of the GFP gene, to allow amplification of the whole GFP ORF by the PCR, and recombination with overlapping identical sequences between the termini of the PCR products and the highly variable, C-terminal region of the PLRV RTD.

Transformation of yeast, recovery of recombinant plasmids, and mobilization to $E$. coli all were done as indicated above. $E$. coli colonies were grown and isolated plasmids were analyzed by sequencing, to confirm the nature of the sequence alterations.

\section{Characterization of PLRV mutants and chimeras.}

Agroinfection was done as described previously by Lee and associates (2002). PLRV and CYDV-RPV concentrations in inoculated and systemically infected leaves were determined by double-antibody sandwich ELISA, using antibodies to the respective virus particles (Barker and Solomon 1990; Rochow and Carmichael 1979). Progeny virus in systemically infected leaves was analyzed by RT-PCR of the viral RNAs in total nucleic acid extracts. The PCR products were sequenced to verify the presence of the introduced mutations or sequence alterations.

\section{ACKNOWLEDGMENTS}

This work was partially supported by NRICGP grant 96-01120 and a grant-in-aid from the Scottish Executive Environment and Rural Affairs Department. We thank D. Smith, L. Lee, and R. Donis for technical assistance and helpful discussion.

\section{LITERATURE CITED}

Barker, H., and Solomon, R. M. 1990. Evidence of simple genetic control in potato of ability to restrict Potato leafroll virus concentration in leaves. Theor. Appl. Genet. 80:188-192.

Botstein, D, and Shortle, D. 1985. Strategies and applications if in vitro mutagenesis. Science 229:1193-1202.

Boyer, J. C., and Haenni, A.-L. 1994. Infectious transcripts and cDNA clones of RNA viruses. Virology 198:415-426.

Bruyère, A., Brault, V., Ziegler-Graff, V., Simonis, M.-T., Van den Heuvel, J. F. J. M., Richards, K., Guilley, H., Jonard, G., and Herrbach, E. 1997. Effect of mutations in the beet western yellows virus readthrough protein on its expression and packaging and on virus accumulation, symptoms, and aphid transmission. Virology 230:323-334.

Domier, L. L., and McCoppin, N. K. 2003. In vivo activity of Rhopalosiphum padi virus internal ribosome entry site. J. Gen. Virol. 84:415419.

Dunø, M., Bendixen, C., Krejci, L., and Thomsen, B. 1999. Targeted deletions created in yeast vectors by recombination excision. Nucleic Acids Res. 27:e1.

Franco-Lara, L. F., McGeachy, K. D., Commandeur, U., Martin, R. R., Mayo, M. A., and Barker, H. 1999. Transformation of tobacco and potato with cDNA encoding the full-length genome of Potato leafroll virus: evidence for a novel virus distribution and host effects on virus multiplication. J. Gen. Virol. 80:2813-2822.

Gardner, R. C., Chonoles, K. R., and Owens, R. A. 1986. Potato spindle tuber viroid infections mediated by the Ti plasmid of Agrobacterium tumefaciens. Plant Mol. Biol. 6:221-228.

Grimsley, N., Hohn, B., Hohn, T., and Walden, R. 1986. Agroinfection, an alternative route for viral-infection of plants by using the Ti plasmid. Proc. Natl. Acad. Sci. U.S.A. 83:3282-3286.

Gunyuzlu, P. L., Hollis, G. F., and Toyn, J. H. 2001. Plasmid construction by linker-assisted homologous recombination in yeast. BioTechniques 31:1246-1250.

Hull, R. 2002. Matthews' Plant Virology, 4th ed. Academic Press, San Diego, CA, U.S.A.

Kawchuk, L., Jaag, H. M., Toohey, K., Martin, R., Rohde, W., and Prüfer, D. 2002. In planta agroinfection by Canadian and German Potato leafroll virus full-length cDNAs. Can. J. Plant Pathol. 24:239-243.

Kitagawa, K., and Abdulle, R. 2002. In vivo site-directed mutagenesis of yeast plasmids using a three-fragment homologous recombination system. BioTechniques 33:288-294.

Kunes, S., Botstein, D., and Fox, M. 1985. Transformation of yeast with linearized plasmid DNA. Formation of inverted dimers and recombinant plasmid. J. Mol. Biol. 184:375-387.

Kunes, S., Ma, H., Overbye, K., Fox, M. S., and Bolstein, D. 1987. Finestructural recombinational analysis of cloned genes using yeast transformation. Genetics 115:73-81.

Lamprecht, S., and Jelkmann, W. 1997. Infectious cDNA clones used to identify strawberry mild yellow edge-associated potexvirus as causal agent of the disease. J. Gen. Virol. 78:2347-2353.

Lee, L., Palukaitis, P., and Gray, S. M. 2002. Host-dependent requirement for the Potato leafroll virus $17-\mathrm{kDa}$ protein in virus movement. Mol. Plant-Microbe Interact. 15:1086-1094.

Leiser, R. M., Ziegler-Graff, V., Reutenauer, A., Herrbach, E., Lemaire, O., Guilley, H., Richards, K., and Jonard, G. 1992. Agroinfection as an alternative to insects for infecting plants with beet western yellows luteovirus. Proc. Natl. Acad. Sci. U.S.A. 89:9136-9140.

Liu, L., and Lomonossoff, G. P. 2002. Agroinfection as a rapid method for propagation of Cowpea mosaic virus-based constructs. J. Virol. Methods 105:343-348.

Liu, Y., Schiff, M., Marathe, R., and Dinesh-Kumar, S. P. 2002. Tobacco Rar1, EDS1 and NPR1/NIM1 like genes are required for $N$-mediated resistance to tobacco mosaic virus. Plant J. 30:415-429.

Ma, H., Kunes, S., Schatz, P. J., and Botstein, D. 1987. Plasmid construction by homologous recombination in yeast. Gene 58:201-216.

Manivasakam, P., Weber, S. C., McElver, J., and Schiestl, R. H. 1995. Micro-homology mediated PCR targeting in Saccharomyces cerevisiae. Nucleic Acids Res. 23:2799-2800.

Nurkiyanova, K. M., Ryabov, E. V., Commandeur, U., Duncan, G. H., Canto, T., Gray, S. M., Mayo, M. A., and Taliansky, M. E. 2000. Tagging Potato leafroll virus with the jellyfish green fluorescent protein gene. J. Gen. Virol. 81:617-626.

Oldenburg, K. R., Vo, K. T., Michaelis, S., and Paddon, C. 1997. Recombination-mediated PRC-directed plasmid construction in vivo in yeast. Nucleic Acids Res. 25:451-452.

Orr-Weaver, T., and Szostak, R. 1985. Fungal recombination. Microbiol. Rev. 49:33-58.

Prüfer, D., Wipf-Scheibel, C., Richards, K., Guilley, H., Lecoq, H., and Jonard, G. 1995. Synthesis of a full-length infectious cDNA of cucurbit aphid-borne yellows virus and its use in gene exchange experiments with structural proteins from other luteoviruses. Virology 214:150-158.

Raymond, C. K., Pownder, T. A., and Sexson, S. L. 1999. General method for plasmid construction using homologous recombination. BioTechniques 26:134-141.

Raymond, C. K., Sims, E. H., and Olson, M. V. 2002. Linker-mediated recombinational subcloning of large DNA fragments using yeast. Genome Res. 12:190-197.

Sambrook, J., and Russell, D. W. 2001. Molecular Cloning: A Laboratory Manual, 3rd ed. Cold Spring Harbor Laboratory Press, Cold Spring Harbor, NY, U.S.A

Satyanarayana, T., Bar-Joseph, M., Mawassi, M., Albiach-Marti, M. R., Ayllón, M. A., Gowda, S., Hilf, M. E., Moreno, P., Garnsey, S. M., and Dawson, W. O. 2001. Amplification of Citrus tristeza virus from a cDNA clone and infection of Citrus trees. Virology 280: 87-96.

Saunders, K., Salim, N., Mali, V. R., Malathi, V. G., Briddon, R., Markham, P. G., and Stanley, J. 2002. Characterisation of Sri Lankan cassava mosaic virus and Indian cassava mosaic virus: Evidence for acquisition of a DNA B component by a monopartite Begomovirus. Virology 293:63-74.

Storici, F., Lewis, L. K., and Resnick, M. A. 2001. In vivo mutagenesis using oligonucleotides. Nat. Biotechnol. 19:773-776.

Sung, P., Trujillo, K. M., Van Komen, S. 2000. Recombination factors of Saccharomyces cerevisiae. Mutat. Res.-Fund. Mol. M. 451:257-275. 